PROCEEDINGS OF THE

AMERICAN MATHEMATICAL SOCIETY

Volume 134, Number 7 , Pages 1973-1983

S 0002-9939(05)08211-0

Article electronically published on December 19, 2005

\title{
ON A CONJECTURE ABOUT MRA RIESZ WAVELET BASES
}

\author{
BIN HAN
}

(Communicated by David R. Larson)

\begin{abstract}
Let $\phi$ be a compactly supported refinable function in $L_{2}(\mathbb{R})$ such that the shifts of $\phi$ are stable and $\hat{\phi}(2 \xi)=\hat{a}(\xi) \hat{\phi}(\xi)$ for a $2 \pi$-periodic trigonometric polynomial $\hat{a}$. A wavelet function $\psi$ can be derived from $\phi$ by $\hat{\psi}(2 \xi):=$ $e^{-i \xi} \overline{\hat{a}(\xi+\pi)} \hat{\phi}(\xi)$. If $\phi$ is an orthogonal refinable function, then it is well known that $\psi$ generates an orthonormal wavelet basis in $L_{2}(\mathbb{R})$. Recently, it has been shown in the literature that if $\phi$ is a $B$-spline or pseudo-spline refinable function, then $\psi$ always generates a Riesz wavelet basis in $L_{2}(\mathbb{R})$. It was an open problem whether $\psi$ can always generate a Riesz wavelet basis in $L_{2}(\mathbb{R})$ for any compactly supported refinable function in $L_{2}(\mathbb{R})$ with stable shifts. In this paper, we settle this problem by proving that for a family of arbitrarily smooth refinable functions with stable shifts, the derived wavelet function $\psi$ does not generate a Riesz wavelet basis in $L_{2}(\mathbb{R})$. Our proof is based on some necessary and sufficient conditions on the $2 \pi$-periodic functions $\hat{a}$ and $\hat{b}$ in $C^{\infty}(\mathbb{R})$ such that the wavelet function $\psi$, defined by $\hat{\psi}(2 \xi):=\hat{b}(\xi) \hat{\phi}(\xi)$, generates a Riesz wavelet basis in $L_{2}(\mathbb{R})$.
\end{abstract}

\section{INTRODUCTION AND MAIN RESUlt}

This paper is about Riesz wavelet bases in $L_{2}(\mathbb{R})$ that are derived from refinable functions. Let $\mathcal{H}$ be a Hilbert space and let $\left\{h_{k}\right\}_{k \in \mathbb{Z}}$ be a sequence of elements in $\mathcal{H}$. We say that $\left\{h_{k}\right\}_{k \in \mathbb{Z}}$ is a Riesz sequence in $\mathcal{H}([9])$ if there exist positive constants $C_{1}$ and $C_{2}$ such that

$$
C_{1} \sum_{k \in \mathbb{Z}}\left|c_{k}\right|^{2} \leqslant\left\|\sum_{k \in \mathbb{Z}} c_{k} h_{k}\right\|^{2} \leqslant C_{2} \sum_{k \in \mathbb{Z}}\left|c_{k}\right|^{2} \quad \forall\left\{c_{k}\right\}_{k \in \mathbb{Z}} \in \ell_{2}(\mathbb{Z}) .
$$

If in addition the linear span of $\left\{h_{k}: k \in \mathbb{Z}\right\}$ is dense in $\mathcal{H}$, then $\left\{h_{k}\right\}_{k \in \mathbb{Z}}$ is said to be a Riesz basis in $\mathcal{H}$. For a function $\psi \in L_{2}(\mathbb{R})$, we say that $\psi$ generates a Riesz wavelet basis in $L_{2}(\mathbb{R})$ if

$$
X(\psi):=\left\{\psi_{j, k}:=2^{j / 2} \psi\left(2^{j} \cdot-k\right): j, k \in \mathbb{Z}\right\}
$$

is a Riesz basis in $L_{2}(\mathbb{R})$.

In order to have a fast wavelet transform, the wavelet function $\psi$ is generally derived from a refinable function via a multiresolution analysis. A function $\phi$ is

Received by the editors October 1, 2004 and, in revised form, February 4, 2005.

2000 Mathematics Subject Classification. Primary 42C20, 41A15, 41A05.

Key words and phrases. Riesz wavelet bases, refinable functions, stability.

This research was supported in part by the Natural Sciences and Engineering Research Council of Canada (NSERC Canada) under Grant G121210654.

(C)2005 American Mathematical Society Reverts to public domain 28 years from publication 
called a refinable function if it satisfies the following refinement equation:

$$
\hat{\phi}(\xi)=\hat{a}(\xi / 2) \hat{\phi}(\xi / 2), \quad \text { a.e. } \xi \in \mathbb{R},
$$

where $\hat{a}$ is a $2 \pi$-periodic measurable function, called the mask for the refinable function $\phi$. The Fourier transform $\hat{f}$ of a function $f \in L_{1}(\mathbb{R})$ is defined to be $\hat{f}(\xi):=\int_{\mathbb{R}} f(t) e^{-i \xi t} d t$ and can be naturally extended to functions in $L_{2}(\mathbb{R})$ and to tempered distributions. A wavelet function $\psi$ is obtained from the refinable function $\phi$ via the following relation:

$$
\hat{\psi}(\xi):=\hat{b}(\xi / 2) \hat{\phi}(\xi / 2), \quad \xi \in \mathbb{R},
$$

for some $2 \pi$-periodic measurable function $\hat{b}$. In many applications such as waveletbased algorithms in image compression, the refinable function $\phi$ is compactly supported and $\hat{a}, \hat{b}$ are $2 \pi$-periodic trigonometric polynomials; so, the wavelet function $\psi$ in (1.4) is also compactly supported. A natural and classical choice of $\hat{b}$ is

$$
\hat{b}(\xi):=e^{-i \xi} \overline{\hat{a}(\xi+\pi)}, \quad \xi \in \mathbb{R} .
$$

It is well known that if $\phi$ is a compactly supported orthogonal refinable function, that is, $\{\phi(\cdot-k): k \in \mathbb{Z}\}$ is an orthonormal system in $L_{2}(\mathbb{R})$, then $\psi$ in (1.4) with $\hat{b}$ given in (1.5) generates an orthonormal wavelet basis in $\left.L_{2}(\mathbb{R})(4]\right)$, which consists of a particular family of Riesz wavelet bases in $L_{2}(\mathbb{R})$.

For a function $\phi$ in $L_{2}(\mathbb{R})$, we say that $\phi$ has stable shifts or the shifts of $\phi$ are stable if $\{\phi(\cdot-k): k \in \mathbb{Z}\}$ is a Riesz sequence in $L_{2}(\mathbb{R})$.

Let $\phi \in L_{2}(\mathbb{R})$ be a refinable function with mask $\hat{a}$ such that the shifts of $\phi$ are stable. There are two interesting fundamental questions about MRA Riesz wavelet bases derived from the refinable function $\phi$.

Question A. For which kind of a $2 \pi$-periodic measurable function $\hat{b}$ does the wavelet function $\psi$ in (1.4) generate a Riesz wavelet basis in $L_{2}(\mathbb{R})$ ?

Question B. For the particular choice of $\hat{b}$ in (1.5), does the wavelet function $\psi$ in (1.4) always generate a Riesz wavelet basis in $L_{2}(\mathbb{R})$ ?

Some sufficient conditions for Question A have been given in the literature 2, 10, 11, 13, 14. More recently, it has been shown in [10] and 6] that for all $B$-spline functions, all refinable interpolating functions with maximum approximation orders, and all pseudo-spline functions (for pseudo-spline functions, see [5]), Question B indeed holds. In other words, so far, for any well-known family of compactly supported univariate refinable functions, Question B holds. Therefore, it is quite appealing to conjecture that Question B holds for all compactly supported refinable functions in $L_{2}(\mathbb{R})$ with stable shifts; Question B has also been implicitly asked in several papers such as [2, 6, 10, 11 and has remained open so far.

In order to answer Question A, in Section 2, we shall provide an almost necessary and sufficient condition on the $2 \pi$-periodic functions $\hat{a}$ and $\hat{b}$ in $C^{\infty}(\mathbb{R})$ such that $\psi$ generates a Riesz wavelet basis in $L_{2}(\mathbb{R})$. Based on part of this result, in Section 3, as the main result of this paper, we shall completely settle Question B by proving the following result:

Main Theorem. For any positive integer $r$, there exists a compactly supported refinable function $\phi$ with $\hat{\phi}(0)=1$ such that

(1) $\hat{\phi}(2 \xi)=\hat{a}(\xi) \hat{\phi}(\xi)$ for a $2 \pi$-periodic trigonometric polynomial $\hat{a}$, 
(2) $\phi$ is a real-valued compactly supported function in $C^{r}(\mathbb{R})$,

(3) the shifts of $\phi$ are stable,

but the wavelet function $\psi$, which is defined by $\hat{\psi}(2 \xi):=e^{-i \xi} \overline{\hat{a}(\xi+\pi)} \hat{\phi}(\xi)$, does not generate a Riesz wavelet basis in $L_{2}(\mathbb{R})$.

In fact, the wavelet function $\psi$ constructed in the above Main Theorem has the properties that $\left\{\psi_{j, k}:-J \leqslant j \leqslant J, k \in \mathbb{Z}\right\}$ is a Riesz sequence for any $J \in \mathbb{N}$ and the span of $\left\{\psi_{j, k}: j, k \in \mathbb{Z}\right\}$ is dense in $L_{2}(\mathbb{R})$. The shifts of the refinable functions $\phi$ in the Main Theorem can even be made linearly independent by a simple perturbation of the masks $\hat{a}$ in our proof of the Main Theorem in Section 3.

We shall prove the Main Theorem in Section 3 and present one example to illustrate the main result in this paper.

\section{RieSZ WAVELET BASES FROM REFINABLE FUNCTIONS}

In this section we shall present an almost necessary and sufficient condition on the $2 \pi$-periodic functions $\hat{a}$ and $\hat{b}$ in $C^{\infty}(\mathbb{R})$ such that the wavelet function $\psi$ in (1.4) generates a Riesz wavelet basis in $L_{2}(\mathbb{R})$.

For $f, g \in L_{2}(\mathbb{R})$, denote $\langle f, g\rangle:=\int_{\mathbb{R}} f(t) \overline{g(t)} d t$, and the bracket product ([12]) is defined to be

$$
[f, g](\xi):=\sum_{k \in \mathbb{Z}} f(\xi+2 \pi k) \overline{g(\xi+2 \pi k)}, \quad \xi \in \mathbb{R} .
$$

Let $\delta$ denote the Dirac sequence on $\mathbb{Z}$ such that $\delta(0)=1$ and $\delta(k)=0$ for all $k \in \mathbb{Z} \backslash\{0\}$. It is well known ([12]) that a function $\phi \in L_{2}(\mathbb{R})$ has stable shifts if and only if there exists a positive constant $C$ such that $C^{-1} \leqslant[\hat{\phi}, \hat{\phi}](\xi) \leqslant C$, a.e. $\xi \in \mathbb{R}$. Moreover, $\langle f, g(\cdot-k)\rangle=\delta(k)$ for all $k \in \mathbb{Z}$ if and only if $[\hat{f}, \hat{g}](\xi)=1$, a.e. $\xi \in \mathbb{R}$, and $\langle f, g(\cdot-k)\rangle=0$ for all $k \in \mathbb{Z}$ if and only if $[\hat{f}, \hat{g}]=0$.

Lemma 1. Let $\phi$ be a refinable function in $L_{2}(\mathbb{R})$ with stable shifts such that $\hat{\phi}(2 \xi)=\hat{a}(\xi) \hat{\phi}(\xi)$, a.e. $\xi \in \mathbb{R}$ for a $2 \pi$-periodic measurable function $\hat{a}$. Define a wavelet function $\psi$ by $\hat{\psi}(2 \xi):=\hat{b}(\xi) \hat{\phi}(\xi)$ for a $2 \pi$-periodic measurable function $\hat{b}$. Assume that

$$
X(\phi, \psi):=\{\phi(\cdot-k): k \in \mathbb{Z}\} \cup\left\{\psi_{j, k}: j \in \mathbb{N} \cup\{0\}, k \in \mathbb{Z}\right\}
$$

is a Riesz basis in $L_{2}(\mathbb{R})$. Then

(i) There exists a positive constant $C$ such that for almost every $\xi \in \mathbb{R}$,

$$
\begin{aligned}
& C^{-1} \leqslant|\hat{a}(\xi)|^{2}+|\hat{a}(\xi+\pi)|^{2} \leqslant C, \quad C^{-1} \leqslant|\hat{b}(\xi)|^{2}+|\hat{b}(\xi+\pi)|^{2} \leqslant C, \\
& \text { and } C^{-1} \leqslant|d(\xi)| \leqslant C \text {, where } \\
& \qquad d(\xi):=\hat{a}(\xi) \hat{b}(\xi+\pi)-\hat{a}(\xi+\pi) \hat{b}(\xi) .
\end{aligned}
$$

(ii) Let $\tilde{\phi}$ and $\tilde{\psi}$ be the unique elements in $L_{2}(\mathbb{R})$ which are determined by

$$
\begin{array}{lll}
\langle\phi, \tilde{\phi}\rangle=1 \quad \text { and } \quad\langle\eta, \tilde{\phi}\rangle=0 & \forall \eta \in X(\phi, \psi) \backslash\{\phi\}, \\
\langle\psi, \tilde{\psi}\rangle=1 \quad \text { and } \quad\langle\eta, \tilde{\psi}\rangle=0 & \forall \eta \in X(\phi, \psi) \backslash\{\psi\} .
\end{array}
$$


Then

$$
\hat{\tilde{\phi}}(2 \xi)=\hat{\tilde{a}}(\xi) \hat{\tilde{\phi}}(\xi) \quad \text { and } \quad \hat{\tilde{\psi}}(2 \xi)=\hat{\tilde{b}}(\xi) \hat{\tilde{\phi}}(\xi), \quad \text { a.e. } \xi \in \mathbb{R},
$$

where

$$
\hat{\tilde{a}}(\xi):=\frac{\overline{\hat{b}(\xi+\pi)}}{\overline{d(\xi)}} \quad \text { and } \quad \hat{\tilde{b}}(\xi):=-\frac{\overline{\hat{a}(\xi+\pi)}}{\overline{d(\xi)}} .
$$

(iii) $X(\tilde{\phi}, \tilde{\psi})$ is the dual Riesz basis of $X(\phi, \psi)$, that is, $X(\tilde{\phi}, \tilde{\psi})$ is a Riesz basis in $L_{2}(\mathbb{R})$ such that for all $k, k^{\prime} \in \mathbb{Z}$ and $j, j^{\prime} \in \mathbb{N} \cup\{0\}$,

$$
\begin{aligned}
& \left\langle\phi_{0, k}, \tilde{\phi}_{0, k^{\prime}}\right\rangle=\delta\left(k-k^{\prime}\right), \quad\left\langle\psi_{j, k}, \tilde{\phi}_{0, k^{\prime}}\right\rangle=0, \\
& \left\langle\phi_{0, k}, \tilde{\psi}_{j^{\prime}, k^{\prime}}\right\rangle=0, \quad\left\langle\psi_{j, k}, \tilde{\psi}_{j^{\prime}, k^{\prime}}\right\rangle=\delta\left(j-j^{\prime}\right) \delta\left(k-k^{\prime}\right) .
\end{aligned}
$$

(iv) There exists a positive constant $C$ such that $|\hat{\tilde{\phi}}(\xi)| \leqslant C,[\hat{\tilde{\phi}}, \hat{\tilde{\phi}}](\xi) \leqslant C$ for almost every $\xi \in \mathbb{R}$ and

$$
\left|\prod_{j=0}^{n} \hat{\tilde{a}}\left(2^{j} \xi\right)\right| \leqslant C, \quad \text { a.e. } \xi \in \mathbb{R}, \quad \forall n \in \mathbb{N} .
$$

Proof. By $\hat{\phi}(2 \xi)=\hat{a}(\xi) \hat{\phi}(\xi)$ and $\hat{\psi}(2 \xi)=\hat{b}(\xi) \hat{\phi}(\xi)$, it is easy to see that

$$
\begin{aligned}
\left(\begin{array}{cc}
{[\hat{\phi}, \hat{\phi}](2 \xi)} & {[\hat{\phi}, \hat{\psi}](2 \xi)} \\
{[\hat{\psi}, \hat{\phi}](2 \xi)} & {[\hat{\psi}, \hat{\psi}](2 \xi)}
\end{array}\right)= & {[\hat{\phi}, \hat{\phi}](\xi)\left(\begin{array}{cc}
|\hat{a}(\xi)|^{2} & \hat{a}(\xi) \overline{\hat{b}(\xi)} \\
\hat{a}(\xi) \hat{b}(\xi) & |\hat{b}(\xi)|^{2}
\end{array}\right) } \\
& +[\hat{\phi}, \hat{\phi}](\xi+\pi)\left(\begin{array}{cc}
|\hat{a}(\xi+\pi)|^{2} & \hat{a}(\xi+\pi) \overline{\hat{b}(\xi+\pi)} \\
\hat{a}(\xi+\pi) \hat{b}(\xi+\pi) & |\hat{b}(\xi+\pi)|^{2}
\end{array}\right) .
\end{aligned}
$$

As a subset of the Riesz sequence $X(\phi, \psi),\left\{\phi_{0, k}, \psi_{0, k}: k \in \mathbb{Z}\right\}$ is also a Riesz sequence. So, there exists a positive constant $C$ such that for almost every $\xi \in \mathbb{R}$,

$$
C^{-1} I_{2} \leqslant\left(\begin{array}{cc}
|\hat{a}(\xi)|^{2}+|\hat{a}(\xi+\pi)|^{2} & \hat{a}(\xi) \overline{\hat{b}(\xi)}+\hat{a}(\xi+\pi) \overline{\hat{b}(\xi+\pi)} \\
\overline{\hat{a}(\xi)} \hat{b}(\xi)+\overline{\hat{a}(\xi+\pi) \hat{b}(\xi+\pi)} & |\hat{b}(\xi)|^{2}+|\hat{b}(\xi+\pi)|^{2}
\end{array}\right) \leqslant C I_{2} .
$$

Consequently, (i) holds since the determinant of the above matrix in the middle term is $|d(\xi)|^{2}$.

Define $\hat{\tilde{\Phi}}(\xi):=\hat{\tilde{a}}(\xi / 2) \hat{\tilde{\phi}}(\xi / 2)$ and $\hat{\tilde{\Psi}}(\xi):=\hat{\tilde{b}}(\xi / 2) \hat{\tilde{\phi}}(\xi / 2)$. By (i) and $\tilde{\phi} \in L_{2}(\mathbb{R})$, we see that $\tilde{\Phi}, \tilde{\Psi} \in L_{2}(\mathbb{R})$. By the definition of $\hat{\tilde{a}}$ and $\hat{\tilde{b}}$ in (2.7), we have

$$
\left(\begin{array}{cc}
\hat{a}(\xi) & \hat{a}(\xi+\pi) \\
\hat{b}(\xi) & \hat{b}(\xi+\pi)
\end{array}\right) \overline{\left(\begin{array}{cc}
\hat{\tilde{a}}(\xi) & \hat{\tilde{b}}(\xi) \\
\hat{\tilde{a}}(\xi+\pi) & \hat{\tilde{b}}(\xi+\pi)
\end{array}\right)}=I_{2}, \quad \text { a.e. } \xi \in \mathbb{R} .
$$

Now we show that (2.4) and (2.5) still hold if $\tilde{\phi}$ and $\tilde{\psi}$ are replaced by $\tilde{\Phi}$ and $\tilde{\Psi}$, respectively.

By (2.4), we have $[\hat{\phi}, \hat{\tilde{\phi}}]=1$. Therefore, by (2.10) and the definition of $\tilde{\Phi}$, we deduce

$$
\begin{aligned}
{[\hat{\phi}, \hat{\tilde{\Phi}}](2 \xi) } & =\hat{a}(\xi) \overline{\tilde{\tilde{a}}(\xi)}[\hat{\phi}, \hat{\tilde{\phi}}](\xi)+\hat{a}(\xi+\pi) \overline{\tilde{\tilde{a}}(\xi+\pi)}[\hat{\phi}, \hat{\tilde{\phi}}](\xi+\pi) \\
& =\hat{a}(\xi) \overline{\tilde{\tilde{a}}(\xi)}+\hat{a}(\xi+\pi) \overline{\tilde{\tilde{a}}(\xi+\pi)}=1
\end{aligned}
$$


and similarly,

$$
\begin{aligned}
{[\hat{\psi}, \hat{\tilde{\Phi}}](2 \xi) } & =\hat{b}(\xi) \overline{\tilde{\tilde{a}}(\xi)}[\hat{\phi}, \hat{\tilde{\phi}}](\xi)+\hat{b}(\xi+\pi) \overline{\tilde{\tilde{a}}(\xi+\pi)}[\hat{\phi}, \hat{\tilde{\phi}}](\xi+\pi) \\
& =\hat{b}(\xi) \overline{\tilde{\tilde{a}}(\xi)}+\hat{b}(\xi+\pi) \overline{\tilde{\tilde{a}}(\xi+\pi)}=0 .
\end{aligned}
$$

That is, $\left\langle\phi_{0, k}, \tilde{\Phi}\right\rangle=\delta(k)$ and $\left\langle\psi_{0, k}, \tilde{\Phi}\right\rangle=0$ for all $k \in \mathbb{Z}$.

For all $j \in \mathbb{N}$ and $k \in \mathbb{Z}$, we have $\left\langle\psi_{j, k}, \tilde{\phi}\right\rangle=\left\langle\psi_{j-1, k}, 2^{-1 / 2} \tilde{\phi}(\cdot / 2)\right\rangle=0$ since $\tilde{\phi}(\cdot / 2) \in V_{0}(\tilde{\phi})$ and by the definition of $\tilde{\phi},\left\langle\psi_{j-1, k}, \tilde{\phi}(\cdot-\ell)\right\rangle=0$ for all $\ell \in \mathbb{Z}$, where $V_{0}(\tilde{\phi})$ denotes the $L_{2}(\mathbb{R})$-closure of the linear span of $\tilde{\phi}(\cdot-\ell), \ell \in \mathbb{Z}$. Consequently, (2.4) holds if $\tilde{\phi}$ is replaced by $\tilde{\Phi}$. Similarly, we can show that (2.5) holds if $\tilde{\psi}$ is replaced by $\tilde{\Psi}$. Therefore, by the uniqueness of $\tilde{\phi}$ and $\tilde{\psi}$, we conclude that $\tilde{\Phi}=\tilde{\phi}$ and $\tilde{\Psi}=\tilde{\psi}$. That is, (2.6) holds and (ii) is true.

By the definition of $\tilde{\phi}$, we deduce that $\left\langle\phi_{0, k}, \tilde{\phi}_{0, k^{\prime}}\right\rangle=\left\langle\phi_{0, k-k^{\prime}}, \tilde{\phi}\right\rangle=\delta\left(k-k^{\prime}\right)$ and $\left\langle\psi_{j, k}, \tilde{\phi}_{0, k^{\prime}}\right\rangle=\left\langle\psi_{j, k-2^{j} k^{\prime}}, \tilde{\phi}\right\rangle=0$ for all $j, j^{\prime} \in \mathbb{N} \cup\{0\}$ and $k, k^{\prime} \in \mathbb{Z}$. By the definition of $\tilde{\psi}$, we have $\left\langle f, \tilde{\psi}_{0, k}\right\rangle=0$ for all $k \in \mathbb{Z}$ and $f \in V_{0}(\phi)$, where $V_{0}(\phi)$ denotes the $L_{2}(\mathbb{R})$-closure of the linear span of $\phi(\cdot-\ell), \ell \in \mathbb{Z}$. Therefore, $\left\langle\phi_{0, k}, \tilde{\psi}_{j^{\prime}, k^{\prime}}\right\rangle=2^{-j^{\prime} / 2}\left\langle\phi\left(2^{-j^{\prime}}.\right), \tilde{\psi}_{0, k^{\prime}-2^{j^{\prime} k}}\right\rangle=0$ since $\phi\left(2^{-j^{\prime}}.\right) \in V_{0}(\phi)$ for all $j^{\prime} \in$ $\mathbb{N} \cup\{0\}$. Similarly, for $j, j^{\prime} \in \mathbb{N} \cup\{0\}$ and $k, k^{\prime} \in \mathbb{Z}$,

$$
\left\langle\psi_{j, k}, \tilde{\psi}_{j^{\prime}, k^{\prime}}\right\rangle= \begin{cases}\left\langle\psi_{j-j^{\prime}, k-2 j-j^{\prime} k^{\prime}}, \tilde{\psi}\right\rangle=0, & \text { if } j>j^{\prime}, \\ \left\langle\psi_{0, k-k^{\prime}}, \tilde{\psi}\right\rangle=\delta\left(k-k^{\prime}\right), & \text { if } j=j^{\prime}, \\ \left\langle\psi_{j-j^{\prime}, 0}, \tilde{\psi}_{0, k^{\prime}-2^{j^{\prime}-j} k}\right\rangle=0, & \text { if } j<j^{\prime},\end{cases}
$$

since $\psi_{j-j^{\prime}, 0} \in V_{0}(\phi)$ for all $j<j^{\prime}$. So, (2.8) holds and (iii) follows from (ii).

By (iii), the shifts of $\tilde{\phi}$ are stable. Therefore, there exists a positive constant $C$ such that $C^{-1} \leqslant[\hat{\tilde{\phi}}, \hat{\tilde{\phi}}](\xi) \leqslant C$, a.e. $\xi \in \mathbb{R}$. By $|\hat{\tilde{\phi}}(\xi)|^{2} \leqslant[\hat{\tilde{\phi}}, \hat{\tilde{\phi}}](\xi) \leqslant C$, we have $|\hat{\tilde{\phi}}(\xi)| \leqslant \sqrt{C}$. Now by (2.6) $), \hat{\tilde{\phi}}\left(2^{n+1} \xi\right)=\hat{\tilde{\phi}}(\xi) \prod_{j=0}^{n} \hat{\tilde{a}}\left(2^{j} \xi\right)$. Therefore,

$$
[\hat{\tilde{\phi}}, \hat{\tilde{\phi}}](\xi) \prod_{j=0}^{n}\left|\hat{\tilde{a}}\left(2^{j} \xi\right)\right|^{2}=\sum_{k \in \mathbb{Z}}\left|\hat{\tilde{\phi}}\left(2^{n+1}(\xi+2 \pi k)\right)\right|^{2} \leqslant[\hat{\tilde{\phi}}, \hat{\tilde{\phi}}]\left(2^{n+1} \xi\right) \leqslant C .
$$

Since $[\hat{\tilde{\phi}}, \hat{\tilde{\phi}}] \geqslant C^{-1}$, we conclude that (2.9) holds.

We say that $\left\{h_{k}\right\}_{k \in \mathbb{Z}}$ is a Bessel sequence in a Hilbert space $\mathcal{H}$ if there exists a positive constant $C_{2}$ such that

$$
\sum_{k \in \mathbb{Z}}\left|\left\langle h, h_{k}\right\rangle\right|^{2} \leqslant C_{2}\|h\|^{2} \quad \forall h \in \mathcal{H} .
$$

Lemma 2. For a function $\psi \in L_{2}(\mathbb{R})$, the following statements are equivalent:

(1) $\left\{\psi_{j, k}: j, k \in \mathbb{Z}\right\}$ is a Bessel sequence in $L_{2}(\mathbb{R})$,

(2) $\left\{\psi_{j, k}: j \geqslant j_{0}, j, k \in \mathbb{Z}\right\}$ is a Bessel sequence in $L_{2}(\mathbb{R})$ for some integer $j_{0}$,

(3) $\left\{\psi_{j, k}: j \leqslant j_{0}, j, k \in \mathbb{Z}\right\}$ is a Bessel sequence in $L_{2}(\mathbb{R})$ for some integer $j_{0}$.

Proof. Note that the inequalities in (1.1) hold for all $\left\{c_{k}\right\}_{k \in \mathbb{Z}} \in \ell_{2}(\mathbb{Z})$ if and only if the inequalities in (1.1) hold for all finitely supported sequences $\left\{c_{k}\right\}_{k \in \mathbb{Z}}$. By $\left\|2^{j / 2} f\left(2^{j}.\right)\right\|=\|f\|$ for all $f \in L_{2}(\mathbb{R})$ and $j \in \mathbb{Z}$, the claim follows directly from the fact that the right-hand inequality in (1.1) holds if and only if (2.11) holds. 
The following result will be needed later.

Proposition 3. Let $\phi \in L_{2}(\mathbb{R})$ be a refinable function with stable shifts such that $\hat{\phi}(2 \xi)=\hat{a}(\xi) \hat{\phi}(\xi)$, a.e. $\xi \in \mathbb{R}$ for a $2 \pi$-periodic measurable function $\hat{a}$. Define a wavelet function $\psi$ by $\hat{\psi}(2 \xi):=\hat{b}(\xi) \hat{\phi}(\xi)$ for a $2 \pi$-periodic measurable function $\hat{b}$. Then $X(\psi):=\left\{\psi_{j, k}: j, k \in \mathbb{Z}\right\}$ is a Riesz basis in $L_{2}(\mathbb{R})$ if and only if $X(\phi, \psi):=\left\{\phi_{0, k}: k \in \mathbb{Z}\right\} \cup\left\{\psi_{j, k}: j \in \mathbb{N} \cup\{0\}, k \in \mathbb{Z}\right\}$ is a Riesz basis in $L_{2}(\mathbb{R})$.

Proof. Suppose that $X(\psi)$ is a Riesz basis in $L_{2}(\mathbb{R})$. Denote $V_{0}$ and $V$ to be the $L_{2}$ closure of the linear spans of the sets $\{\phi(\cdot-k): k \in \mathbb{Z}\}$ and $\left\{\psi_{j, k}: j<0, j, k \in \mathbb{Z}\right\}$, respectively. By $\hat{\psi}(2 \xi)=\hat{b}(\xi) \hat{\phi}(\xi)$ and [1, Corollary 3.8], we have $V_{0}=V$. Since $X(\psi)$ is a Riesz basis in $L_{2}(\mathbb{R})$, we see that $\left\{\psi_{j, k}: j<0, j, k \in \mathbb{Z}\right\}$ is a Riesz basis in $V_{0}$. Since $\phi$ has stable shifts, we see that $\left\{\phi_{0, k}: k \in \mathbb{Z}\right\}$ is also a Riesz basis in $V_{0}$. Since $X(\psi)$ is a Riesz basis in $L_{2}(\mathbb{R})$, now it is easy to deduce that $X(\phi, \psi)$ must be a Riesz basis in $L_{2}(\mathbb{R})$.

Conversely, suppose that $X(\phi, \psi)$ is a Riesz basis in $L_{2}(\mathbb{R})$. By Lemma $X(\tilde{\phi}, \tilde{\psi})$ is the dual Riesz basis of $X(\phi, \psi)$, where $\tilde{\phi}$ and $\tilde{\psi}$ are defined in Lemma 1. Now by (2.8), it is easy to verify that $\left\langle\psi_{j, k}, \tilde{\psi}_{j^{\prime}, k^{\prime}}\right\rangle=\delta\left(j-j^{\prime}\right) \delta\left(k-k^{\prime}\right)$ for all $j, j^{\prime}, k, k^{\prime} \in$ $\mathbb{Z}$. By Lemma 2 both $X(\psi)$ and $X(\tilde{\psi})$ are Bessel sequences in $L_{2}(\mathbb{R})$. By the biorthogonality of $X(\psi)$ and $X(\tilde{\psi})$, we deduce that $X(\psi)$ and $X(\tilde{\psi})$ are Riesz sequences in $L_{2}(\mathbb{R})$. Since the functions $\phi$ and $\tilde{\phi}$ are refinable and the linear spans of $X(\phi, \psi)$ and $X(\tilde{\phi}, \tilde{\psi})$ are dense in $L_{2}(\mathbb{R})$, it follows from a standard argument in multiresolution analysis that the linear spans of $X(\psi)$ and $X(\tilde{\psi})$ are dense in $L_{2}(\mathbb{R})$. Therefore, $X(\psi)$ and $X(\tilde{\psi})$ are Riesz bases in $L_{2}(\mathbb{R})$.

For a function $\phi \in L_{2}(\mathbb{R})$, the difference operator $\nabla_{t}: L_{2}(\mathbb{R}) \mapsto L_{2}(\mathbb{R})$ is defined to be

$$
\nabla_{t} \phi:=\phi-\phi(\cdot-t), \quad t \in \mathbb{R} .
$$

Corollary 4. Under the same conditions and notation as in Lemma 1, then the shifts of $\tilde{\phi}$ are stable and consequently,

$$
\lim _{n \rightarrow \infty} 2^{n} \int_{-\pi}^{\pi}\left|\left(1-e^{-i \xi}\right)^{m}\right|^{2} \prod_{j=0}^{n-1}\left|\hat{\tilde{a}}\left(2^{j} \xi\right)\right|^{2} d \xi=0 \quad \forall m \in \mathbb{N} .
$$

If in addition we assume that $\lim _{n \rightarrow \infty}[\hat{\phi}, \hat{\phi}]\left(2^{-n} \xi\right)=\lim _{n \rightarrow \infty} \hat{\phi}\left(2^{-n} \xi\right)=1$ a.e. $\xi \in$ $\mathbb{R}$, then $\lim _{n \rightarrow \infty} \hat{\tilde{\phi}}\left(2^{-n} \xi\right)=1$ and $\hat{\tilde{\phi}}(\xi)=\prod_{j=1}^{\infty} \hat{\tilde{a}}\left(2^{-j} \xi\right)$ a.e. $\xi \in \mathbb{R}$.

Proof. By (iii) of Lemma 1, the shifts of $\tilde{\phi}$ are stable and (2.6) holds. Since $\hat{\tilde{\phi}}(2 \xi)=$ $\hat{\tilde{a}}(\xi) \hat{\tilde{\phi}}(\xi)$, we deduce that $\hat{\tilde{\phi}}\left(2^{n} \xi\right)=\hat{\tilde{\phi}}(\xi) \prod_{j=0}^{n-1} \hat{\tilde{a}}\left(2^{j} \xi\right)$ and consequently,

$$
\begin{aligned}
\int_{\mathbb{R}}\left|\left(1-e^{-i \xi}\right)^{m} \hat{\tilde{\phi}}\left(2^{n} \xi\right)\right|^{2} d \xi & =\int_{\mathbb{R}}|\hat{\tilde{\phi}}(\xi)|^{2}\left|\left(1-e^{-i \xi}\right)^{m}\right|^{2} \prod_{j=0}^{n-1}\left|\hat{\tilde{a}}\left(2^{j} \xi\right)\right|^{2} d \xi \\
& =\int_{-\pi}^{\pi}[\hat{\tilde{\phi}}, \hat{\tilde{\phi}}](\xi)\left|\left(1-e^{-i \xi}\right)^{m}\right|^{2} \prod_{j=0}^{n-1}\left|\hat{\tilde{a}}\left(2^{j} \xi\right)\right|^{2} d \xi .
\end{aligned}
$$


Note that the Fourier transform of $2^{-n}\left[\nabla_{2^{-n}}^{m} \tilde{\phi}\right]\left(2^{-n} \cdot\right)$ is $\left(1-e^{-i \xi}\right)^{m} \hat{\tilde{\phi}}\left(2^{n} \xi\right)$. Therefore, we have

$$
\begin{aligned}
2^{n} \int_{-\pi}^{\pi}[\hat{\tilde{\phi}}, \hat{\tilde{\phi}}](\xi)\left|\left(1-e^{-i \xi}\right)^{m}\right|^{2} & \prod_{j=0}^{n-1}\left|\hat{\tilde{a}}\left(2^{j} \xi\right)\right|^{2} d \xi=2^{n} \int_{\mathbb{R}}\left|\left(1-e^{-i \xi}\right)^{m} \hat{\tilde{\phi}}\left(2^{n} \xi\right)\right|^{2} d \xi \\
& =2^{-n} \int_{\mathbb{R}}\left|\left(\left[\nabla_{2^{-n}}^{m} \tilde{\phi}\right]\left(2^{-n} \cdot\right)\right)^{\wedge}(\xi)\right|^{2} d \xi \\
& =2 \pi 2^{-n}\left\|\left[\nabla_{2^{-n}}^{m} \tilde{\phi}\right]\left(2^{-n} \cdot\right)\right\|^{2}=2 \pi\left\|\nabla_{2^{-n}}^{m} \tilde{\phi}\right\|^{2}
\end{aligned}
$$

Since the shifts of $\tilde{\phi}$ are stable, there exists a positive constant $C$ such that $[\hat{\tilde{\phi}}, \hat{\tilde{\phi}}] \geqslant$ $C^{-1}>0$. Hence,

$$
\begin{aligned}
2^{n} \int_{-\pi}^{\pi}\left|\left(1-e^{-i \xi}\right)^{m}\right|^{2} & \prod_{j=0}^{n-1}\left|\hat{\tilde{a}}\left(2^{j} \xi\right)\right|^{2} d \xi \\
& \leqslant C 2^{n} \int_{-\pi}^{\pi}[\hat{\tilde{\phi}}, \hat{\tilde{\phi}}](\xi)\left|\left(1-e^{-i \xi}\right)^{m}\right|^{2} \prod_{j=0}^{n-1}\left|\hat{\tilde{a}}\left(2^{j} \xi\right)\right|^{2} d \xi \\
& =2 \pi C\left\|\nabla_{2^{-n}}^{m} \tilde{\phi}\right\|^{2} .
\end{aligned}
$$

Since $\tilde{\phi} \in L_{2}(\mathbb{R})$ and $m \geqslant 1$, we have $\lim _{n \rightarrow \infty}\left\|\nabla_{2^{-n}}^{m} \tilde{\phi}\right\|=0$. Therefore, we conclude that (2.13) holds.

By (iii) of Lemma 1, $[\hat{\tilde{\phi}}, \hat{\tilde{\phi}}] \in L_{\infty}(\mathbb{R})$ and $[\hat{\tilde{\phi}}, \hat{\phi}]=1$. So,

$$
1-\hat{\tilde{\phi}}(\xi) \overline{\hat{\phi}(\xi)}=[\hat{\tilde{\phi}}, \hat{\phi}](\xi)-\hat{\tilde{\phi}}(\xi) \overline{\hat{\phi}(\xi)}=\sum_{k \in \mathbb{Z} \backslash\{0\}} \hat{\tilde{\phi}}(\xi+2 \pi k) \overline{\hat{\phi}(\xi+2 \pi k)} .
$$

By the Cauchy-Schwarz inequality, we have

$$
\left|\sum_{k \in \mathbb{Z} \backslash\{0\}} \hat{\tilde{\phi}}(\xi+2 \pi k) \overline{\hat{\phi}(\xi+2 \pi k)}\right|^{2} \leqslant[\hat{\tilde{\phi}}, \hat{\tilde{\phi}}](\xi)\left([\hat{\phi}, \hat{\phi}](\xi)-|\hat{\phi}(\xi)|^{2}\right) .
$$

Therefore,

$$
\left|1-\hat{\tilde{\phi}}\left(2^{-n} \xi\right) \overline{\hat{\phi}\left(2^{-n} \xi\right)}\right| \leqslant\|[\hat{\tilde{\phi}}, \hat{\tilde{\phi}}]\|_{L_{\infty}(\mathbb{R})}\left([\hat{\phi}, \hat{\phi}]\left(2^{-n} \xi\right)-\left|\hat{\phi}\left(2^{-n} \xi\right)\right|^{2}\right) .
$$

Consequently, by our assumption that $\lim _{n \rightarrow \infty}[\hat{\phi}, \hat{\phi}]\left(2^{-n} \xi\right)=\lim _{n \rightarrow \infty} \hat{\phi}\left(2^{-n} \xi\right)=1$, it follows from the above inequality that $\lim _{n \rightarrow \infty} \hat{\tilde{\phi}}\left(2^{-n} \xi\right) \overline{\hat{\phi}\left(2^{-n} \xi\right)}=1$. Now by $\lim _{n \rightarrow \infty} \hat{\phi}\left(2^{-n} \xi\right)=1$, we conclude that $\lim _{n \rightarrow \infty} \hat{\tilde{\phi}}\left(2^{-n} \xi\right)=1$ a.e. $\xi \in \mathbb{R}$.

By (2.6), we have $\hat{\tilde{\phi}}(\xi)=\hat{\tilde{\phi}}\left(2^{-n} \xi\right) \prod_{j=1}^{n} \hat{\tilde{a}}\left(2^{-j} \xi\right)$. Hence, $\hat{\tilde{\phi}}(\xi)=\prod_{j=1}^{\infty} \hat{\tilde{a}}\left(2^{-j} \xi\right)$ by $\lim _{n \rightarrow \infty} \hat{\tilde{\phi}}\left(2^{-n} \xi\right)=1$ a.e. $\xi \in \mathbb{R}$.

In the following, let us generalize a quantity $\nu_{2}(\hat{a})$ defined in [8, Page 61] and [7, Page 697]. For a $2 \pi$-periodic measurable function $\hat{a}$, we define

$$
\rho(\hat{a}):=\inf _{m \in \mathbb{N}} \limsup _{n \rightarrow \infty}\left(\int_{-\pi}^{\pi}\left|\left(1-e^{-i \xi}\right)^{m}\right|^{2} \prod_{j=0}^{n-1}\left|\hat{a}\left(2^{j} \xi\right)\right|^{2} d \xi\right)^{1 / n}
$$

and

$$
\nu_{2}(\hat{a}):=-\left[1+\log _{2} \rho(\hat{a})\right] / 2 .
$$


For a $2 \pi$-periodic trigonometric polynomial $\hat{a}$, the quantity $\nu_{2}(\hat{a})$ plays a very important role in characterizing the convergence of a cascade algorithm in a Sobolev space and in characterizing the Sobolev smoothness of the refinable function with mask $\hat{a}$ (see [8] and the references therein). When $\hat{a}$ is a $2 \pi$-periodic trigonometric polynomial, the quantity $\nu_{2}(\hat{a})$ can be numerically computed by finding the spectral radius of a certain finite matrix (see [2, 4, 7] and the references therein). For $2 \pi$-periodic measurable functions $\widehat{a_{1}}$ and $\widehat{a_{2}}$, if $\left|\widehat{a_{1}}(\xi)\right| \leqslant\left|\widehat{a_{2}}(\xi)\right|$ a.e. $\xi \in \mathbb{R}$, then it follows from the definition in (2.16) that $\nu_{2}\left(\widehat{a_{2}}\right) \leqslant \nu_{2}\left(\widehat{a_{1}}\right)$.

Now we have the following result.

Corollary 5. Let $\phi$ be a refinable function in $L_{2}(\mathbb{R})$ with stable shifts such that $\hat{\phi}(2 \xi)=\hat{a}(\xi) \hat{\phi}(\xi)$, a.e. $\xi \in \mathbb{R}$ for a $2 \pi$-periodic measurable function $\hat{a}$. Define $\psi$ by $\hat{\psi}(2 \xi):=\hat{b}(\xi) \hat{\phi}(\xi)$ for a $2 \pi$-periodic measurable function $\hat{b}$. If $\nu_{2}(\hat{\tilde{a}})<0$, where $\hat{\tilde{a}}$ is defined in (2.7), then $\psi$ does not generate a Riesz wavelet basis in $L_{2}(\mathbb{R})$.

Proof. Suppose that $\psi$ generates a Riesz wavelet basis in $L_{2}(\mathbb{R})$. By Proposition 3 and Corollary 4, (2.13) must be true. Now it is easy to see that (2.13) implies $\rho(\hat{\tilde{a}}) \leqslant 1 / 2$ and therefore, we must have $\nu_{2}(\hat{\tilde{a}}) \geqslant 0$, which is a contradiction to our assumption $\nu_{2}(\hat{\tilde{a}})<0$. So, $\psi$ does not generate a Riesz wavelet basis in $L_{2}(\mathbb{R})$.

Now we have the following result on MRA Riesz wavelet bases.

Theorem 6. Let $\hat{a}$ and $\hat{b}$ be two $2 \pi$-periodic functions in $C^{\infty}(\mathbb{R})$ such that $\hat{a}(0)=1$ and $\hat{b}(0)=0$. Define

$$
\hat{\phi}(\xi):=\prod_{j=1}^{\infty} \hat{a}\left(2^{-j} \xi\right) \quad \text { and } \quad \hat{\psi}(\xi):=\hat{b}(\xi / 2) \hat{\phi}(\xi / 2) .
$$

If $\phi \in L_{2}(\mathbb{R})$ has stable shifts and $\psi$ generates a Riesz wavelet basis in $L_{2}(\mathbb{R})$, under the additional assumption that $\lim _{n \rightarrow \infty}[\hat{\phi}, \hat{\phi}]\left(2^{-n} \xi\right)=1$ a.e. $\xi \in \mathbb{R}$, then

(1) $d \in L_{\infty}(\mathbb{R})$ and $1 / d \in L_{\infty}(\mathbb{R})$, where $d(\xi):=\hat{a}(\xi) \hat{b}(\xi+\pi)-\hat{a}(\xi+\pi) \hat{b}(\xi)$,

(2) $[\hat{\phi}, \hat{\phi}] \in L_{\infty}(\mathbb{R})$ and $[\hat{\tilde{\phi}}, \hat{\tilde{\phi}}] \in L_{\infty}(\mathbb{R})$,

(3) both $X(\psi)$ and $X(\tilde{\psi})$ are Bessel sequences in $L_{2}(\mathbb{R})$,

(4) $\nu_{2}(\hat{a}) \geqslant 0$ and $\nu_{2}(\hat{\tilde{a}}) \geqslant 0$,

where $\hat{\tilde{a}}$, $\hat{\tilde{b}}$ are defined in (2.7) and

$$
\hat{\tilde{\phi}}(\xi):=\prod_{j=1}^{\infty} \hat{\tilde{a}}\left(2^{-j} \xi\right) \quad \text { and } \quad \hat{\tilde{\psi}}(\xi):=\hat{\tilde{b}}(\xi / 2) \hat{\tilde{\phi}}(\xi / 2) .
$$

Conversely, if (1), (2), (3) hold and

$\left(4^{\prime}\right) \nu_{2}(\hat{a})>0$ and $\nu_{2}(\hat{\tilde{a}})>0$,

then $\phi \in L_{2}(\mathbb{R})$ has stable shifts and $\psi$ generates a Riesz wavelet basis in $L_{2}(\mathbb{R})$.

Proof. By Proposition 3 and Corollary 4, the function $\tilde{\phi}$, defined in Lemma 1 is the same function as defined in (2.18). Now (1), (2), (3) and (4) follow directly from Lemma 1 and Corollary [5.

Conversely, it has been proved in [11, Theorem 4.2] that $\left(4^{\prime}\right)$ implies $\phi, \tilde{\phi} \in L_{2}(\mathbb{R})$ and $[\hat{\phi}, \hat{\tilde{\phi}}]=1$. By $(2)$, we deduce that the shifts of $\phi$ are stable. Now by a standard argument as in [3], X( $\psi$ ) and $X(\tilde{\psi})$ form a pair of biorthogonal wavelet bases in 
$L_{2}(\mathbb{R})$. So, $\psi$ generates a Riesz wavelet basis in $L_{2}(\mathbb{R})$. Also see [2, 14] for the case that the Fourier coefficients of $\hat{a}$ and $\hat{b}$ have exponential decay.

We mention that the condition $\hat{a}, \hat{b} \in C^{\infty}(\mathbb{R})$ in Theorem 6 can be replaced by a much weaker condition, but the presentation here is simpler.

\section{Proof of the Main Theorem}

In this section, we prove the Main Theorem in Section 1 and provide an example.

Proof of the Main Theorem. Let $\hat{a}$ be a $2 \pi$-periodic trigonometric polynomial such that

(i) $\hat{a}(0)=1, \hat{a}(\pi)=0$, and

$$
h:=\frac{|\hat{a}(2 \pi / 3)|}{|\hat{a}(2 \pi / 3)|^{2}+|\hat{a}(2 \pi / 3+\pi)|^{2}} \frac{|\hat{a}(4 \pi / 3)|}{|\hat{a}(4 \pi / 3)|^{2}+|\hat{a}(4 \pi / 3+\pi)|^{2}}>1 .
$$

(ii) Its associated compactly supported refinable function $\phi$ lies in $L_{2}(\mathbb{R})$ and the shifts of $\phi$ are stable, where $\hat{\phi}(\xi):=\prod_{j=1}^{\infty} \hat{a}\left(2^{-j} \xi\right)$.

Let $\hat{b}(\xi):=e^{-i \xi} \overline{\hat{a}(\xi+\pi)}$ and $\hat{\psi}(\xi):=\hat{b}(\xi / 2) \hat{\phi}(\xi / 2)$. In the following, we show that $\psi$ cannot generate a Riesz wavelet basis in $L_{2}(\mathbb{R})$.

Let $\hat{\tilde{a}}$ and $\hat{\tilde{b}}$ be defined in (2.7). Then by calculation, we have

$$
\hat{\tilde{a}}(\xi)=\frac{\hat{a}(\xi)}{|\hat{a}(\xi)|^{2}+|\hat{a}(\xi+\pi)|^{2}} .
$$

Let $\tilde{\phi}, \tilde{\psi}$ be defined in (2.18). Now we show that $\hat{\tilde{\phi}} \notin L_{\infty}(\mathbb{R})$. For any nonnegative integer $n$, we observe that $2^{n} 2 \pi / 3 \equiv 2 \pi / 3$ or $4 \pi / 3 \bmod 2 \pi$. By (3.1), we have $|\hat{\tilde{a}}(2 \pi / 3) \hat{\tilde{a}}(4 \pi / 3)|=h>1$. Therefore, we conclude that

$$
\left|\prod_{j=0}^{2 n-1} \hat{\tilde{a}}\left(2^{j} 2 \pi / 3\right)\right|=|\hat{\tilde{a}}(2 \pi / 3) \hat{\tilde{a}}(4 \pi / 3)|^{n}=h^{n} \quad \forall n \in \mathbb{N} .
$$

Since the shifts of $\phi$ are stable and $\phi$ is compactly supported, there exists $k_{0} \in \mathbb{Z}$ such that $\hat{\phi}\left(2 \pi / 3+2 \pi k_{0}\right) \neq 0$. By $|\hat{a}(\xi)|^{2}+|\hat{a}(\xi+\pi)|^{2} \neq 0$ for all $\xi \in \mathbb{R}$, it follows from the definition of $\hat{\tilde{\phi}}$ in (2.18) that $\hat{\tilde{\phi}}(\xi)=0$ if and only if $\hat{\phi}(\xi)=0$. Therefore, $\hat{\tilde{\phi}}\left(2 \pi / 3+2 \pi k_{0}\right) \neq 0$. By (3.2) and $\hat{\tilde{\phi}}(\xi)=\hat{\tilde{a}}(\xi / 2) \hat{\tilde{\phi}}(\xi / 2)$, we deduce that

$$
\begin{aligned}
\left|\hat{\tilde{\phi}}\left(2^{2 n}\left(2 \pi / 3+2 \pi k_{0}\right)\right)\right| & =\left|\hat{\tilde{a}}\left(2^{2 n-1} 2 \pi / 3\right) \cdots \hat{\tilde{a}}(2 \pi / 3)\right|\left|\hat{\tilde{\phi}}\left(2 \pi / 3+2 \pi k_{0}\right)\right| \\
& =h^{n}\left|\hat{\tilde{\phi}}\left(2 \pi / 3+2 \pi k_{0}\right)\right| \quad \forall n \in \mathbb{N} .
\end{aligned}
$$

Since $\hat{\tilde{\phi}}\left(2 \pi / 3+2 \pi k_{0}\right) \neq 0$ and $h>1$, we conclude that

$$
\lim _{n \rightarrow \infty}\left|\hat{\tilde{\phi}}\left(2^{2 n}\left(2 \pi / 3+2 \pi k_{0}\right)\right)\right|=\infty \text {. }
$$

Since $\hat{\tilde{\phi}}$ is a continuous function, we have $\hat{\tilde{\phi}} \notin L_{\infty}(\mathbb{R})$. Note that $\hat{a}(\pi)=0$ implies $\hat{\phi}(2 \pi k)=0$ for all $k \in \mathbb{Z} \backslash\{0\}$. Since $\phi$ is compactly supported, $[\hat{\phi}, \hat{\phi}]$ is a $2 \pi$-periodic trigonometric polynomial and therefore, we have $\lim _{\xi \rightarrow 0}[\hat{\phi}, \hat{\phi}](\xi)=1$ by $\hat{\phi}(0)=1$ and $\hat{\phi}(2 \pi k)=0$ for all $k \in \mathbb{Z} \backslash\{0\}$.

Suppose that $\psi$ indeed generates a Riesz wavelet basis in $L_{2}(\mathbb{R})$. Since the shifts of $\phi$ are stable, by Proposition 3. Lemma 1 holds. But (3.2) is a contradiction to 
(2.9) in Lemma 1. Or, by Theorem 6] we must have $\hat{\tilde{\phi}} \in L_{\infty}$, a contradiction to our conclusion $\hat{\tilde{\phi}} \notin L_{\infty}(\mathbb{R})$. So, $\psi$ cannot generate a Riesz wavelet basis in $L_{2}(\mathbb{R})$.

To complete the proof, let us show that such desirable masks $a$ satisfying (i) and (ii) do exist. Let $m$ be a positive integer. Let $c$ be a $2 \pi$-periodic trigonometric polynomial with real coefficients such that

$$
c(0)=1, \quad c(2 \pi / 3)=c(4 \pi / 3)<2^{-m} \quad \text { and } \quad 0<c(\xi) \leqslant 1 \quad \forall \xi \in \mathbb{R} .
$$

Clearly, there are many $2 \pi$-periodic trigonometric polynomials $c$ such that (3.3) holds. Take $\hat{a}(\xi):=2^{-m}\left(1+e^{-i \xi}\right)^{m} c(2 \xi)$. Clearly, $\hat{a}(0)=1, \hat{a}(\pi)=0$, and

$$
|\hat{\tilde{a}}(\xi)|=\frac{|\hat{a}(\xi)|}{|\hat{a}(\xi)|^{2}+|\hat{a}(\xi+\pi)|^{2}}=\frac{|\cos (\xi / 2)|^{m}}{c(2 \xi)\left[\cos ^{2 m}(\xi / 2)+\sin ^{2 m}(\xi / 2)\right]} \geqslant \frac{|\cos (\xi / 2)|^{m}}{c(2 \xi)} .
$$

Therefore,

$$
h=|\hat{\tilde{a}}(2 \pi / 3) \hat{\tilde{a}}(4 \pi / 3)| \geqslant \frac{\cos ^{2 m}(\pi / 3)}{c(4 \pi / 3) c(8 \pi / 3)}=\frac{1}{\left[2^{m} c(2 \pi / 3)\right]^{2}}>1 .
$$

So, (i) holds. By the definition of $\hat{\phi}$ and $|\hat{a}(\xi)| \leqslant|\cos (\xi / 2)|^{m}$, we have

$$
|\hat{\phi}(\xi)| \leqslant\left|\frac{\sin (\xi / 2)}{\xi / 2}\right|^{m} \quad \forall \xi \in \mathbb{R} \text {. }
$$

Hence, we have $\phi \in C^{m-2}(\mathbb{R})$. By the definition of $\hat{a}$, we see that $\hat{\phi}(\xi)=0$ for some $\xi \in \mathbb{R}$ if and only if $\xi=2 \pi k$ for some $k \in \mathbb{Z} \backslash\{0\}$. So, (ii) holds.

Finally, let us present one example to illustrate the main result in this paper.

Example 7. Let $\hat{a}(\xi):=\left|1+e^{-i \xi}\right|^{2}\left[3 e^{2 i \xi}-2 e^{i \xi}+10-2 e^{-i \xi}+3 e^{-2 i \xi}\right] / 48$. Using [7. Algorithm 2.1], by calculation, we have $\nu_{2}(\hat{a}) \approx 1.87994>0$ and therefore, the compactly supported refinable function $\phi$ with mask $\hat{a}$ belongs to $L_{2}(\mathbb{R})$. Define

$$
\begin{gathered}
\hat{\mathrm{a}}(\xi):=\left|1+e^{-i \xi}\right|^{2}[226+76 \cos (\xi)-244 \cos (2 \xi)-47 \cos (3 \xi)+126 \cos (4 \xi) \\
-55 \cos (5 \xi)+20 \cos (6 \xi)-6 \cos (7 \xi)] / 384 .
\end{gathered}
$$

Then $\nu_{2}(\stackrel{\hat{a}}{)} \approx 0.41911$ and the compactly supported refinable function $\dot{\phi}$ with mask $\hat{a}$ lies in $L_{2}(\mathbb{R})$. Since $\overline{\hat{a}(\xi)} \hat{a}(\xi)+\overline{\hat{a}(\xi+\pi)} \hat{a}(\xi+\pi)=1$, we have $[\hat{\phi}, \hat{\phi}]=1$ by $\nu_{2}(\hat{a})>0$ and $\nu_{2}(\hat{a})>0$. So, the shifts of $\phi$ are linearly independent and therefore, stable. However, by calculation, we have $-0.83695 \leqslant \nu_{2}(\hat{\tilde{a}}) \leqslant-0.29600<0$ and $\hat{\tilde{a}}(2 \pi / 3)=\hat{\tilde{a}}(4 \pi / 3)=24 / 17>1$. By Theorem 6] the wavelet function $\psi$, which is defined by $\hat{\psi}(2 \xi):=e^{-i \xi} \hat{\hat{a}(\xi+\pi)} \hat{\phi}(\xi)$, does not generate a Riesz wavelet basis in $L_{2}(\mathbb{R})$.

\section{ACKNOWLEDGMENT}

The author would like to thank Professor Rong-Qing Jia at the University of Alberta and Professor Zuowei Shen at the National University of Singapore for several discussions on MRA Riesz wavelet bases, which inspired this work. The author also thanks the anonymous referee for helpful suggestions that improved the presentation of this paper. 


\section{REFERENCES}

[1] M. Bownik, Riesz wavelets and generalized multiresolution analyses, Appl. Comput. Harmon. Anal. 14 (2003), 181-194. MR.1984546 (2004d:42057)

[2] A. Cohen and I. Daubechies, A new technique to estimate the regularity of refinable functions, Rev. Mat. Iberoamericana 12 (1996), 527-591. MR.1402677(97g:42025)

[3] A. Cohen, I. Daubechies and J.C. Feauveau, Biorthogonal bases of compactly supported wavelets, Comm. Pure Appl. Math. 45 (1992), 485-560. MR.1162365(93e:42044)

[4] I. Daubechies, Ten Lectures on Wavelets, CBMS-NSF Series in Applied Mathematics, SIAM, Philadelphia, 1992. MR1162107 (93e:42045)

[5] I. Daubechies, B. Han, A. Ron, and Z. Shen, Framelets: MRA-based constructions of wavelet frames, Appl. Comput. Harmon. Anal. 14 (2003), 1-46. MR1971300 (2004a:42046)

[6] B. Dong and Z. W. Shen, Pseudo-splines, wavelets and framelets, preprint, (2004).

[7] B. Han, Computing the smoothness exponent of a symmetric multivariate refinable function, SIAM J. Matrix Anal. Appl. 24 (2003), 693-714. MR.1972675 (2004b:42078)

[8] B. Han, Vector cascade algorithms and refinable function vectors in Sobolev spaces, J. Approx. Theory. 124 (2003), 44-88. MR.2010780 (2004h:42034)

[9] D. G. Han and D. R. Larson, Frames, bases and group representations, Mem. Amer. Math. Soc. 147, No. 697, (2000). MR1686653(2001a:47013)

[10] B. Han and Z. W. Shen, Wavelets with short support, preprint, (2003).

[11] B. Han and Z. W. Shen, Wavelets from the Loop scheme, J. Fourier Anal. Appl., to appear.

[12] R. Q. Jia and C. A. Micchelli, Using the refinement equation for the construction of prewavelets II: Power of two, Curves and Surfaces (P.J. Laurent, A. Le Méhauté and L. L. Schumaker, eds.), Academic Press, New York, (1991), 209-246. MR.1123739 (93e:65024)

[13] R. Q. Jia, J. Z. Wang, and D. X. Zhou, Compactly supported wavelet bases for Sobolev spaces, Appl. Comput. Harmon. Anal. 15 (2003), 224-241. MR2010944 (2004h:42042)

[14] R. Lorentz and P. Oswald, Criteria for hierarchical bases in Sobolev spaces, Appl. Comput. Harmon. Anal. 8 (2000), 32-85. MR.1734847(2001h:46051)

Department of Mathematical and Statistical Sciences, University of Alberta, Edmonton, Alberta, Canada T6G 2G1

E-mail address: bhan@math.ualberta.ca

$U R L:$ http://www.ualberta.ca/ bhan 